Fecha de recepción: diciembre 2012 Fecha de aceptación: septiembre 2013 Versión final: septiembre 2014

\section{Metodologías del diseño en la promoción de aprendizaje organizacional}

Paula Visoná ${ }^{\star}$ y Giulio Palmitessa ${ }^{\star *}$

\begin{abstract}
Resumen: El diseño como disciplina fenomenológica surge en el escenario actual como mecanismo de creación de relaciones sinérgicas entre empresas y universidades. Muchas veces estas relaciones tienen sus bases en una revisión de los procesos internos de las organizaciones. En este contexto, las metodologías del diseño pueden establecer nuevos parámetros de aprendizaje organizacional, creando formas de participación fluida y creativa a través de sectores y competencias. Para ilustrar esta perspectiva, presentamos un modelo de aprendizaje basado en la educación a través de la práctica del proyecto reflexivo utilizando la herramienta del Workshop, notable instrumento metodológico de aprendizaje creativo del Diseño Estratégico. De esta manera, este trabajo presenta el estudio de casos de experiencias proyectuales de los Workshops llevados a cabo en colaboración con la marca de calzado de plástico Melissa. Los resultados fueron soluciones innovadoras, tanto en el ámbito de la educación organizacional, como en el campo de aplicación de nuevos parámetros de comprensión del diseño para los diseñadores de la marca.
\end{abstract}

Palabras clave: aprendizaje organizacional - diseño estratégico - innovación - metodologías del diseño - workshops.

[Resúmenes en inglés y portugués en las páginas 156-157]

${ }^{(*)}$ Master en Diseño Universidad del Vale de lo Río de los Sinos (Unisinos). Licenciada en Moda por la Universidad de Caxias do Sul (UCS). Coordinadora de los cursos de Posgrado en Diseño Estratégico y Diseño de Moda. Unisinos. Es profesora en las carreras de Moda y Diseño en Unisinos.

${ }^{(*)}$ Coordinador de Design Center da Escola de Design da UNISINOS. Profesor de Diseño y metodologias de poryecto en Bacharelado y técnico en Diseño en la misma institución. Master of Sciences Furniture e textile Design, Universitá Politecnico di Milano, profesor de Pós-Graduação da UNISINOS. Autor de diversos artículos em revistas del sector de diseño de mobiliário. Coordenador General de la muestra Italian Genius Now Brasil para Sudamérica. 


\section{Introducción. Articulaciones entre empresa y academia.}

Es conocida la importancia de construir relaciones sinérgicas entre las empresas y las academias. Se trata de buscar caminos para desarrollar "búsquedas aplicadas" promoviendo la revisión de paradigmas incorporados primero por las organizaciones empresariales y luego viralizados en los mercados. En el estudio presentado en este texto, se tiene como objetivo mostrar formas de promover tanto las relaciones sinérgicas arriba mencionadas como los momentos de práctica y reflexión proyectual que, por su naturaleza propia, estimulan la revisión de hábitos y costumbres incorporados por sectores internos de las empresas. Por lo tanto, se parte de la interpretación del diseño como proceso a fin de observar mecanismos para la implantación de la cultura de proyecto dentro en una empresa específica, en este caso Grendene, en el marco de Melissa Academy.

La aproximación de la empresa Gredene -a través de su marca Melissa- al abordaje proyectual complejo, se desarrolló según el proyecto articulado por la Escuela de Diseño Unisinos que utiliza el concepto de Diseño Estratégico para promover nuevas formas de pensar, dirigir, hacer y practicar Diseño.

\section{El proyecto Melissa Academy}

La empresa Grendene nació en la ciudad de Farroupilha, en el interior de Rio Grande do Sul, en 1971. Inicialmente se ocupaba de la producción de embalajes plásticos. Con el tiempo y gracias al empeño y determinación de sus fundadores Alexandre y Pedro Grendene Bartelle la organización se fue transformando en la mayor sede de fabricación de calzado del país.

El cambio del foco productivo desde los embalajes hacia el calzado de plástico se desarrolló a través de la interpretación de una debilidad detectada en el mercado de la moda hacia fines de la década de los setenta. En ese momento nació la marca Melissa. Melissa se consolidó como ícono de la moda y es referente para fashionistas brasileños, teenagers americanas y consumidores de los cinco continentes. A través del concepto Full Plastic, la marca promovió el reposicionamiento de la empresa en los mercados del Diseño y de la moda, tanto nacionales como internacionales.

Es importante destacar que a partir de la entrada de la marca Melissa al mercado de la moda, Grendene incorporó varios aspectos inherentes al campo de la moda en su estrategia como empresa. Esas transformaciones generaron cambios hacia adentro y hacia afuera de la empresa. De acuerdo con Tidd, Bessant y Pavitt (2005) la innovación es un proceso que se establece a partir del cambio.

¿Que entendemos por “innovación”? Básicamente, estamos hablando de cambio, y esto puede tomar varias formas. [...] La innovación de productos -cambio en las cosas (productos, servicios) que ofrece una empresa; innovación de proceso -cambios en la forma en que productos/servicios son creados y entregados; Innovación de posición -cambia el contexto en 
que productos/servicios son introducidos; paradigma de la innovación cambios en los modelos mentales subyacentes que guían lo que la empresa hace (TIDD, Bessant y Pavitt, 2005, p. 30).

Durante mucho tiempo, la marca Melissa basó su estrategia en la innovación de productos y procesos ofreciendo objetos y servicios que ayudaron a mantener su posición de liderazgo. Sin embargo, en los últimos años -después de todo, la marca tiene una historia de más de tres décadas- los administradores y diseñadores interpretaron la trascendencia de la innovación y alinearon esa perspectiva a los cambios identificados en el comportamiento del consumo. Para comprender mejor este aspecto, merece la pena introducir consideraciones del filósofo Vilém Fluser. De acuerdo con Flusser el comportamiento social actual está alineado con lo que caracterizó como Homo Ludens, el hombre que experimenta con los sentidos:

El nuevo hombre ya no es una persona de acciones concretas, sino un performer (Spieler): Homo ludens en lugar de Homo faber. Para él, la vida ya no es un drama y se convirtió en un espectáculo. Ya no se trata de acciones, sino de sensaciones. El nuevo hombre no quiere tener que hacer, quiere experimentar (Flusser, 2007, p. 58).

De ese modo, los diseñadores de la marca Melissa comprendieron la importancia de atender esa demanda: las experiencias de los sentidos a través de los objetos producidos por la marca. A partir de ese momento surgirán varios proyectos especiales. La mayoría de estos proyectos fueron coordinados por el Departamento de Investigación y desarrollo de productos de la empresa a cargo de su Director Edson Matsuo.

En este contexto surgió en 2010 el proyecto de Melissa Academy. Este emprendimiento se caracterizó por ser una plataforma de colaboración proyectual, cuyo objetivo es estimular el interés y la creatividad de los futuros profesionales del Diseño en el mundo. El propósito del proyecto es fomentar el desarrollo de nuevas formas de pensar, trabajar y crear con plástico y además desarrollar un diseño curatorial para acercar al gran público las novedades que están siendo lanzadas en el sector. Melissa Academy intenta cumplir ese objetivo mediante el desarrollo de asociaciones entre la marca, las escuelas y las universidades de Diseño y moda.

La primera asociación se efectuó con la renombrada escuela de Arte y Diseño de Ginebra (Haute École d'Art et Design), Head Gèneve. De acuerdo con Edson Matsuo, creador del proyecto: "El tema principal no fue el diseño de un zapato, sino un walking object y un body accessory. Fue una experiencia fantástica: 24 alumnos de varias nacionalidades de los cursos de Diseño de modas y joyas produjeron excelentes resultados". Matsuo junto a Fernando Mascaro, consultor de Diseño sustentable de Melissa, y profesores de la institución administraron workshops y clases para los participantes. De entre las propuestas presentadas por los alumnos fueron elegidas tres. Los proyectos fueron lanzados al mercado en ediciones limitadas, siendo incorporados a la colección de productos presentados por Melissa en enero de 2011 en Fashion Rio (una de las más significativas semanas de la moda en Brasil). 
Un poco antes de esto, todavía en el año 2010, la marca comenzó un proceso de aproximación con la escuela de Diseño Unisinos, situada en la ciudad de Porto Alegre, al sur de Brasil. Matsuo y Mascaro fueron los articuladores de esa aproximación que al principio intentó alcanzar el mismo objetivo propuesto a Head Gèneve. Pero a medida que se fueron realizando reuniones entre los coordinadores del proyecto Melissa Academy y los profesores de la escuela de Diseño UNISINOS, se plantearon otros objetivos.

\section{La escuela de Diseño UNISINOS}

La escuela de Diseño de la UNISINOS (EDU, como es comúnmente conocida), es el resultado de una colaboración con Poli.Design, un consorcio del Politécnico di Milano. Este proyecto tuvo como objetivo la implantación de la primera escuela de investigación del estado de Rio Grande do Sul, creando así un ambiente propicio para el desarrollo de competencias en diseño a partir de un acuerdo de colaboración y transferencia de tecnologías desde la perspectiva del diseño estratégico y la innovación orientadas al diseño.

Desde el inicio, el proyecto intentó interpretar de manera innovadora el complejo sistema de la educación tratando de crear, desarrollar, planificar y aplicar el concepto de Design School. ${ }^{1}$ Cabe de destacar la visión estratégica del diseño que el Prof. Dr. Francesco Zurlo ${ }^{2}$ propone a través de la introducción al pensamiento complejo del filósofo Edgar Morin, alineando con esa misma esa perspectiva. En ese contexto se entrevé un abordaje orgánico del diseño, entendiéndolo a partir de ese enfoque, como un sistema complejo de relaciones. En las propias palabras de Zurlo: "Un sistema abierto que incluye puntos de vista diferentes” (Zurlo, 2006, pp. 142-144).

En la metodología propuesta para la escuela de Diseño UNISINOS, el enfoque complejo del DESIGN se desarrolla a través del diseño estratégico, que por otro lado, se organiza a través del sistema-producto. El mismo autor define al sistema-producto como "un conjunto integrado de producto, servicio, comunicación y distribución a través del cual una empresa se desenvuelve en el mercado".

Es un sistema de interfaces a través de las cuales la organización se presenta ante la sociedad y da forma a la propia estrategia" (Zurlo, 2010).

Profesores e investigadores de ambas instituciones crearon los orígenes de un entorno integrado entre los tres ámbitos que caracterizan el modus operandi de la EDU.

La primera de estas áreas tiene como objetivo la construcción del conocimiento científico y aplicado (proyectos immateria, Tedx Unisinos - Innovación en la educación, revista científica Strategic Design Research Journal, etc..), esta área es intensamente estimulada por el Programa de Posgrado en Diseño, principalmente a través de los resultados de producción científica de los investigadores de la EDU.

La segunda área se caracteriza por compromiso con la enseñanza de los cursos lato sensu, Stricto sensu, Maestría, Cursos de Grado, Especialización y Extensión. La oferta formativa diferenciada por niveles y por profundidad de conocimiento, objetiva y la enseñanza "práctica y reflexiva para desarrollar habilidades profesionales" (SCHÖN, 2000).

La tercera área es el Design Center que se constituyó como un centro de investigación y desarrollo en Diseño. Este centro tiene como objetivo primario promover y difundir la 
cultura del proyecto en la sociedad. Con este propósito se establecieron sociedades entre empresas, órganos de gobierno y entidades sin fines de lucro en diferentes niveles, dejando en evidencia una clara relación sistema-mercado. Estas asociaciones activan el aspecto estratégico del sistema-producto de la escuela de Diseño UNISINOS. Las actividades se concretan a través de la realización de Workshops realizados en los cursos de Maestría, Especialización y de Grado de la Escuela.

\section{El Design Center de la EDU}

"la escuela de diseño es un espacio transdisciplinar de investigación, práctica e integración para la promoción y difusión de la cultura del diseño. En sus actividades explora tanto el pensar cuanto el hacer del diseño a través de la innovación, la creatividad y el enfoque estratégico" (Escuela de Diseño UNISINOS). ${ }^{3}$

El Design Center de la EDU es el ambiente catalizador que relaciona el sistema-mercado y el sistema-producto a través de la aplicación del proyecto, interpretando este proceso como un descubrimiento. ${ }^{4}$ El centro de innovación orientado al diseño de la UNISINOS (Design Center) sistematiza los procesos de concepción y aprendizaje de la creatividad para la generación de nuevos productos-servicios. ${ }^{5}$ Este centro propone difundir la cultura del proyecto contemporáneo y la innovación con eje en el diseño. Esas prerrogativas están alineadas con el mercado. Por tanto aplican las conclusiones y los resultados en diversas organizaciones.

Dentro del estudio del caso presentado, el proyecto Melissa Academy se configura como un ambiente de sensemaking 6 activado a través de un proceso de enactment (WEICK, 1995) y desarrollado en el Design Center de la EDU. Durante este proceso se analizan las articulaciones de los actores involucrados, es decir, profesores y alumnos de la EDU, y profesionales de Melissa. Durante este proceso, la actividad de los sujetos no se rige estrictamente por las metas de concepción de productos como resultado. Según la interpretación de los profesores de la Escuela de Design UNISINOS esas articulaciones deberían darse en los Workshops promovidos para interpretar el briefing (que también se fue construido en un modo de colaboración, o sea, entre Matsuo, Mascaro y profesores de la institución). En ese sentido, Melissa Academy también podría contemplar otra meta: la de establecer caminos para la revisión de etapas relativas a la proyección de productos de la empresa, por lo tanto, de promoción de espacios para interpretar la perspectiva de aprendizaje organizativo.

\section{Innovación como modelo cultural}

Para Celaschi (2007) proyectar un producto nuevo o rediseñar un objeto ya existente implica una clara acción de innovación del producto, sin embargo como los productos/ servisicios son cada vez más intangibles para las exigencias y cambios de las ofertas en el mercado (por ejemplo, el taxi o el coche compartido en lugar de la adquisición de un 
automóvil), se crean ofertas de servicios o experiencias que están más relacionadas con la innovación de los procesos que con la innovación de los productos en sí. Como señala el autor, "alterar la forma del bien significa modificar el proceso de comunicación, de distribución, de interfaces, de materiales, de asistencia, etc." (Celaschi, 2007).

Este enfoque se muestra interesante porque evidencia la innovación como proceso de transformación de modelos culturales en las organizaciones. Por lo tanto, se puede abordar de la siguiente forma: la primera fase es externa a la entidad y propone una mirada en el sistema de consumo donde una organización debe cuidar la dimensión cualitativa de la mercadería (Celaschi, 2000) e integrar en la innovación las reglas que modificaran la conceptualización del producto y sus visiones "ampliadas"7 (LEVITT, 1980); la segunda, es relativa a los criterios de análisis del éxito de las organizaciones en el mercado ${ }^{8}$ donde la innovación es el principal índice de evaluación y no el crecimiento o el beneficio. Y la tercera fase está relacionada directamente con la comprensión del modelo cultural del proceso de innovación relacionado a "estructuras y dispositivosque van más allá del entorno económico y de organización” (Borba y Reyes, 2009). Esa práctica está bien sintetizada por los autores, que interpretan a la innovación a partir de cuatro focos: en resultado económico, en la estrategia, en la creatividad y en la tolerancia al error:

1. [...] Estos cuatro puntos son los que caracterizan al diseño estratégico, dado que sobre esta perspectiva el diseño presupone: resultado económico generando valor para la empresa; una perspectiva estratégica viendo el producto en todo su ciclo de vida; el proceso creativo como fuente de innovación, que presupone un método y sistematización; y la tolerancia al error, que considera la búsqueda de alternativas a partir de diferentes escenarios. (Borba y Reyes, 2009).

2. A partir de las argumentaciones tejidas por los autores, es posible comprender que la primera implicación está ligada a modificaciones en la cultura empresarial, implementando costumbres y hábitos direccionados a la búsqueda de la innovación. En ese sentido, es preciso entender cómo suceden esos procesos. Sin duda, el Diseño Estratégico en su definición de sistema abierto que incluye puntos de vista diferentes, privilegia herramientas metodológicas que potencializan la implantación de procesos destinados a la innovación en las organizaciones. Uno de esos procesos se caracteriza por la construcción colaborativa del briefing, contemplando la posibilidad de reposicionamiento y dimensionándolo como contra- briefing (Celaschi, 2007), que identifica en el modelo estratégico de actuar (Zurlo, 2010) como un diferencial único.

Según Zurlo (2010) "El diseño, como la cultura de proyecto en general, tiene una extensión natural en relación a lo nuevo. Por eso el Diseño no puede distinguirse de la innovación. Diseño es innovación”. Esto se relaciona, principalmente, con la interpretación del diseño como la cultura del proyecto contemporáneo, con foco en el proceso y no en el producto como dimensión estratégica (La carta di Torino, 2008)9. Este planteo es premisa fundamental para posicionar a la "ciencia del diseño" que Donald Shön trata como "epistemología de la práctica" y se caracteriza por la capacidad de producir conocimientos a través de las prácticas. En este contexto, la definición del diseño como una disciplina 
fenomenológica, es el abordaje de la práctica del Diseño en la Escuela de Diseño Unisinos: “... la observación de la realidad del proyecto para trazar reglas generales y principios generales que todavía evolucionan juntos y de forma continua sobre el punto de vista adoptado para el marco de referencia del proyecto" (Bertola, 2006).

La lógica de la innovación definida por el diseño, revela un terreno fértil en el proceso para identificar la importancia del desarrollo continuo de normas y dinámicas que permitan trazar nuevos caminos para alcanzar dicha la innovación.

Promover nuevos procesos es el mecanismo que permite que coincidan los enfoques del Diseño Estratégico (Borba y Reyes, 2009) y el de Melissa Academy. Si el objetivo del proyecto Melissa Academy es fomentar el desarrollo de nuevas formas de pensar, se determina que el modelo de la cultura de la innovación propuesto por la EDU cumple el objetivo de promover la ruptura de algunos paradigmas internos de la empresa Grendene. En este sentido, coinciden las perspectivas apuntadas por los fundadores del Proyecto Melissa Academy, con la marca Melissa designada para recorrer este camino.

El modelo de este caso de estudio no está destinado a la innovación económica, sino más bien una reflexión sobre los hábitos y costumbres construidas por los diseñadores de la marca. En este contexto, la innovación es evidente a partir de los modelos culturales, siendo la sociedad escuela/empresa, una forma de aplicar transformaciones en las prácticas y dinámicas de hacer diseño. Por lo tanto, hablamos de un nuevo modelo de diseño basado en el disfrute del proceso.

\section{Workshop como método en la busca de caminos de innovación}

Según Phillippe Comte (2010), un workshop de diseño se puede definir como un momento de ejercicios creativos y ensayos, donde herramientas y competencias son adecuadas a un determinado contexto. Este concepto se fundamenta a partir de una problemática -un briefing- en el que son identificados objetivos y metas a ser cumplidas, en un periodo preestablecido. Debido a que es un proceso complejo de proyectar, nuevos ajustes pueden efectuarse mediante la creación de un workshop, que puede tener una duración variable, generalmente de dos días a una semana (Comte, 2010, pp. 52-55).

Dentro de las prácticas didácticas del curso de Bacharel en diseño de la EDU, los workshops se conforman momentos de inmersión creativa, con el fin de señalar el camino hacia la innovación a través de técnicas variadas. Este diseño dinámico puede llevar a la formulación de escenarios proyectivos, conceptos de diseño, o proposiciones concretas. La finalidad es contemplar el brief de proyecto en todas sus dimensiones. (Scalestsky, 2008, p. 1135). Para Cautela (2007), un workshop de diseño envuelve tres grupos: los diseñadores, los tutores y los clientes. En este estudio específico el cliente se ha configurado como la marca Melissa, los diseñadores eran estudiantes de la Licenciatura en Diseño UNISINOS y los profesores que estuvieron presentes durante toda la realización de de los Workshops, fueron los tutores. Para hacer frente a las intenciones presentadas por los coordinadores del proyecto Melissa Academy, se realizaron dos workshops en la EDU durante el año 2011. El primero fue en febrero, teniendo como objetivo principal la generación de ideas para nuevos productos para la marca Melissa. Ya el segundo workshop realizado en el mes de 
abril de 2011, se ocupó de presentar articulaciones entre dimensiones en torno a esos productos, tales como servicio, comunicación y experiencias. Ambos fueron viabilizados por el Design Center de la escuela, vía graduación.

Debido a su importancia, este estudio se ocupará de relatar los procesos que componen el primer workshop realizado en el mes de febrero de 2011. Esta experiencia de diseño buscó contemplar dos objetivos distintos: a) promover la innovación a nivel de producto mediante la propuesta de conceptos, y b) de presentar caminos para la revisión de herramientas y métodos de proyección a los profesionales de la empresa Grendene. Esta segunda perspectiva se desarrolla a través de la implicación de los directivos y diseñadores de la marca Melissa durante la realización de los Workshops. Para entonces, y gracias a la relación establecida entre profesores, estudiantes y profesionales, fue posible contemplar el propósito de la revisión de las costumbres y los hábitos incorporados a la lógica de hacer y de pensar diseño de la empresa con el fin de lograr algunos paradigmas de la gestión de proyectos incorporados por la organización.

\section{Workshop I. De lo común a lo a extraordinario}

Después de algunas reuniones con los coordinadores del proyecto Melissa Academy, se destacó la importancia de tener no solo un briefing, sino también una guía temática para los proyectos. En este sentido se definió que el briefing del workshop debía estar relacionado al objetivo fundamental del proyecto Melissa Academy, a direccionar los asuntos, a definir las herramientas y los caminos a ser desarrollados Así se llego a la síntesis:

Briefing: nuevas forma de pensar, trabajar y crear con plástico.

Temática: de ordinario a extraordinario.

A partir de estas resoluciones, se iniciaron conversaciones entre profesores de la EDU y diseñadores de la empresa con el fin de entender cómo serán elaborados esas orientaciones, o sea, ordinario y extraordinario. En ese enfoque, se comprende que ordinario se relacionaría a la cuestión del calzado de plástico como objeto que promueve la movilidad. Ya extraordinario, al contenido simbólico que podría ser desarrollado a partir de cada concepto de producto. Entre tanto, la temática del workshop se concentró en dos dimensiones ampliamente discutidas en los workshops celebrados en la UNISINOS: tangible e intangible. A partir de este momento, se pasó a definir los contenidos, competencias y herramientas que serían utilizadas a lo largo del primer workshop. En lo que respecta al contenido, surgieron dos direcciones principales:

Subversión y transgresión de la manera de generar nuevos sentidos: el diseño como generador de sentido

Para contemplar la perspectiva de subversión y transgresión, se trató de relacionar esos conceptos a la forma de los objetos y a las dinámicas de desplazamiento de funciones para 
interpretar nuevos significados. En este contexto, esta aproximación se basó en los argumentos ofrecidos por Benjamin (1994) y Foucault (2006).

A partir de Benjamin (1994), se puede considerar que la transgresión, que funciona como dispositivo operante para el consumo de bienes simbólicos en la moda, también puede funcionar como factor de distracción. También de acuerdo con el autor, la distracción es interpretada como una postura de entretenimiento frente a la obra de arte. Por analogía con las obras de arte, tomar una postura de entretenimiento delante de objetos de moda supuestamente transgresores opera en el límite, en el borde debido al acto inherentemente subversivo para el objeto y sus características estéticas.

En cuanto a Foucault, la transgresión no se caracteriza por ser una actitud de negación generalizada,

..., sino como una afirmación de que no dice nada en plena ruptura de la transitividad. El reto no es el esfuerzo del pensamiento para negar acciones o valores, es el gesto que trae cada uno de ellos a sus límites [...]. Allí, dentro de los límites transgredidos, repercute la defensa. (Foucault, 2006, p. 34).

Partiendo de esas consideraciones, se buscó relacionar ejemplos que permitiesen la comprensión de la importancia de transgredir los límites formales de los objetos por la encarada por la marca Melissa, agregando la dimensión de ruptura de algunos patrones de características proyectuales principalmente estéticas y después simbólicas.

Para ello, se decidió construir una perspectiva más cercana de la transgresión operada por el arte, el trabajo La Fuente (1917), obra de Marcel Duchamp, como ejemplo. El trabajo, que se caracteriza por ser un urinario para expresar la subjetividad del artista, sirvió para expresar los preceptos dadaístas, la comunicación al público en general, el contenido de la subversión de la estética, la artística y la moral propuesto por el movimiento en el inicio del siglo XX.

Este contenido se inició con las discusiones tejidas durante el Workshop, como un mecanismo para estimular la búsqueda de contribuciones simbólicas que pudieran responder principalmente a la temática del workshop.

\section{El diseño como un generador de sentido}

El primer trabajo de cambio de paradigmas en la transgresión de los cánones estéticos de un producto hizo que los participantes del workshop comenzaron a definir los límites de la dimensión simbólica a ser diseñada.

La creación de elementos de discusión y el malestar en los estudiantes dejó un ambiente muy activo y dispuesto a responder a la interpretación del briefing. Este proceso fue muy importante para que el grupo pudiera entender el campo de acción, la libertad y la autonomía proyectual, evitando así las restricciones pre-establecidas y distanciarse más allá del ámbito de aplicación propuesto por la empresa (Best, 2006). Se creo así, un ambiente de significados compartidos acerca del briefing que inició la construcción de los elementos intangibles del proyecto que, según Zurlo (2010) son típicas del diseño estratégico; “[...] 
el diseño estratégico se manifiesta, [...], como esa actividad de proyecto completando la activación de la acción estratégica dentro de las estructuras organizadas" (Zurlo, 2010). Además, según el pensamiento del autor, si la estrategia según la visión del diseño es un proceso de creación de sentido, se interpreta una relación entre la creación de significado y la capacidad del diseño para crearse a sí mismo. Según Zingale (2008), quien también hace referencia a Zurlo, la habilidad del diseño para crear efectos de sentido resulta en la capacidad de atraer y motivar a los diferentes actores. Estos actores no son necesariamente los consumidores finales, (lógica del Human Center Design), sino todos los actores vinculados al sistema-producto de la empresa "[...] accionistas, líderes de mercado, [...]” (Zurlo, 2010).

En este contexto, entendimos que los estudiantes debían comenzar a individualizar los elementos intangibles relacionados con el sistema-producto Melissa y se seleccionaron opciones para la presentación y discusión de varios ejemplos de productos de diseño. Uno de ellos es la silla Favela de 1990, de los hermanos Campana, producida por en Río Grande do Sul, pensada por diseñadores brasileños y comercializada por una empresa símbolo del Made in Italy, EDRA. La silla fue inspirada en la favela da Rocinha, en Río de Janeiro, y tiene la particularidad de estar construida con trozos de de madera OSB, de forma caótica. Como relatan los hermanos Campana en una entrevista: "queríamos reproducir la manera como se forma una favela" (Garcia y Hermes, 2009 en www. casa.abril.com.br) El éxito de este producto define muy bien la capacidad del grupo italiano, formado por industrias capaces de individualizar alrededor del mundo objetos y diseñadores con historias y aquellas las habilidades que se alinean con las identidades de sus empresas. Un significado que va más allá de la localización de la producción. Los usuarios reciben varios efectos de sentido viendo esta silla, y conociendo su historia confluyen efectos que se superponen de manera convergente (Zurlo, 2010).

El workshop logró impulsar, de manera objetiva, la exploración de la construcción de sentido -sensemaking- antes de comenzar a proyectar.

\section{Aprendizaje y resultados}

De acuerdo a los pasos y a las herramientas que se utilizaron en la realización de Workshops patrocinados por la EDU para dar cabida a los objetivos del proyecto Melissa, podemos sintetizar los siguientes resultados.

Los resultados obtenidos en el primer Workshop fueron ideas para nuevos productos para la marca. Pero como ya se mencionó, en ese momento de la inmersión creativa también se trató de promover la revisión de algunas de las prácticas para diseñar incorporadas por la empresa -y por lo tanto- por los diseñadores de la marca.

Esta promoción y revisión del aprendizaje se aplica desde los años 90 como la motivación en el entorno empresarial a través del aprendizaje organizacional (Crossan et al., 1999). Según el autor, la construcción del conocimiento pasa a través de los diferentes modos de aprendizaje, ya sea de manera individual, colectiva o de la misma organización. Este modelo dirigido a un modelo colectivo de aprendizaje organizacional tiene que pasar por una fase de revisión de las prácticas institucionalizadas de un grupo, para luego ser 
incorporados a las nuevas dinámicas de la empresa. (Senge, 1990; Argyris, 1991). En consecuencia, hemos tratado de combinar profesionales y estudiantes en equipos de proyectos (cinco en total). Estos equipos utilizaron un ámbito de aplicación con el fin de obtener prototipos rápidos que permitieran ver los conceptos de diseño que se estaban proponiendo. La dinámica de interacción entre los profesionales de la Grendene y los alumnos de la EDU, contempla llevar a cabo prácticas de revisión incorporadas por la organización promoviendo la observación de algunos paradigmas relacionados con la visión del diseño de la empresa. En esta experiencia de inmersión creativa a través de workshop, los profesionales de la empresa se relacionan con herramientas que fomentan una visión sistémica del proyecto, principalmente de la observación, el trabajo de campo y la búsqueda Blue Sky.

A partir de este entendimiento, los gestores del sector de desarrollo de producto de la Grendene-que también participan en algunos momentos puntuales de los workshops- se conectan con la importancia de comprender el diseño como una práctica abierta, que permite la interacción de varios actores, relacionando conocimientos y puntos de vista de una hibridez inherentemente interdisciplinar. En este caso, la inmersión creativa proporcionada por el Workshop sirvió como llave del vehículo relacional contemplando la conexión entre el cambio y el aprendizaje (Garvin, 1993; Kim, 1993; Marquadt, 1996).

La dinámica desarrollada a lo largo principalmente del primer workshop, acaba generando una revisión de algunos métodos utilizados para la obtención de soluciones de productos de marca Melissa atendiendo, por lo tanto, a la necesidad de promover el aprendizaje de nuevas prácticas de diseño. En este sentido, el primer workshop de la asociación entre EDU y Melissa proporcionó mecanismos para la generación de nuevas técnicas de proyección, y las mismas fueron repetidas dentro de la empresa Grendene después de la finalización del mismo.

En la relación entre aprendizaje y mejoras (Garvin, 1993) analizó el objetivo del aprendizaje organizacional con el fin de promover rutas de acceso a las prácticas innovadoras y a las dinámicas relacionadas con la proyección de los futuros productos (tanto de la marca Melissa, como de otras marcas desarrolladas por Grendene).

Los conceptos de producto diseñados por los equipos de proyecto servirán para trasmitir los resultados y también presentarán tanto respuestas al briefing de workshop, como a la temática propuesta. Como la perspectiva del proyecto Melissa Academy es el lanzamiento en el mercado nacional e internacional de los productos pensados en asociación con escuelas de moda y diseño del mundo, no es posible presentar imágenes o comentarios sobre los resultados proyectuales alcanzados en los Workshops. Solamente nos cabe comentar que habrá un lanzamiento de un producto Melissa Academy + escuela de diseño UNISINOS en el año 2012. El lanzamiento del producto expresa la importancia de promover un diálogo entre la empresa y la escuela, llevando a la luz pública algunas de las dinámicas de participación de estos organismos con el fin de que puedan ser replicados en otros sectores a través principalmente las perspectivas de fomento de la innovación abierta y la creación en colaboración.

El segundo workshop patrocinado por las uniones entre la EDU y Grendene trató de pensar el sistema de relaciones de cada concepto de diseño a raíz del workshop anterior. Los resultados obtenidos en esta segunda inmersión creativa de sistema-producto se basan en conceptos previamente seleccionados. En este contexto, hemos tratado de coordinar el 
servicio, la comunicación y la experiencia de cada proyecto de workshop I. Fueron cinco en total. Como se lanzarán dos proyectos (a través de la marca Melissa), también se presentará el sistema-producto diseñado para articular las dimensiones expandidas del producto. Mientras tanto la compañía posibilita que sus prácticas de gestionar y hacer diseño se interrelacionen para diseñar un abordaje complejo practicado a través de Diseño Estratégico en la escuela de diseño UNISINOS. La marca Melissa es la plataforma relacional que permite la hibridación, lo que puede -y debe, de acuerdo con los profesionales de la empresa- ser replicada en otras marcas de Grendene.

\section{Notas}

1. “[...] de una intensa colaboración entre los profesores e investigadores brasileños (UNISINOS) e italianos, que buscan crear un espacio académico, conceptual, cultural y de formación en que creación y gestión actúen de manera íntegra y sinérgica para el desenvolvimiento de la sociedad. Desde su creación, la Escuela -llamada cariñosamente EDU- se caracterizó por la integración entre las áreas académicas y la comunidad, por la innovación como un proceso que genera valor para la sociedad y la internacionalización de sus profesores y estudiantes como sus proyectos e iniciativas" (Liberali, 2008, p. 30).

2. El Prof. Dr. Francesco Zurlo fue director de POLI.Design e investigador. Contribuyó intensamente en el desarrollo teórico de la disciplina del diseño "estratégico" y cofundó la Escuela de Diseño UNISINOS. Hace 14a ediciones el Prof. Dr. Zurlo F. es Director del primer Máster en Diseño Estratégico del Politécnico di Milano, y realizó el primer curso de especialización en Diseño Estratégico de la Escuela de Diseño UNISINOS.

3. Definición de la Escuela de Diseño UNISINOS desarrollada en la actividad de workshop de la Junta Directiva en el segundo semestre de 2011. Esta definición construyó el reposicionamiento estratégico de la EDU.

4."proviene del Latin projectu, participio pasado del verbo projecere, que significa poner en marcha hacia adelante" (Veiga, 2001, p. 12).

5. Definimos como productos-servicios los resultados de la producción de conocimientos, enseñanzas e investigaciones aplicadas.

6. Para Karl Weick, la producción de sentido es lo que produce la organización, definida como un sistema abierto, que hace sentido para todos sus actores y es ordenada por el proceso constante de construcción de entidades que se mueven entre las subjetividades (Karl Weick, 1995).

7. "producto ampliado" (Levitt, 1980), con una óptica de "productos, más los servicios que la empresa ofrece, más los servicios que los clientes no esperan".

8. En la última década, es interesante destacar cómo diferentes autores construirán y direccionarán a los medios económicos nuevos modelos de análisis de la organización. Las clasificaciones que las revistas como Forbes (2011), o Businessweek (2006) presentan una variación sustancial en los índices de evaluación de una empresa y de su modelo de innovación (Dyer, Gregersen, Christensen, 2011). 
9. ICSID ha nombrado a Torino Primera Capital Mundial del Diseño porque es una ciudad que ha sabido rediseñarse con un renovado rol internacional en la era posindustrial, sobre todo gracias a los procesos y a los métodos del diseño. El primer encuentro de investigadores y estudiosos de los procesos y métodos del diseño se llevó a cabo en Torino el 12 de julio de 2008 en ocasión del Foro de la Conferencia Internacional de las Escuelas de Doctorado en Diseño "Changing the Change". Por este motivo decidimos llamar "Carta di Torino" al manifesto que llevamos a los miembros del Foro Permanente que aquí se constituye y al que proponemos adherir y difundir a la comunidad internacional del Diseño".

\section{Bibliografía}

Argyris, C. (1992). Organizational Learning. Massachusetts: Blackwell Publishers.

Benjamin, W. (1994). Magia e técnica, arte e política: ensaios sobre literatura e história da cultura. 7. ed. São Pulo: Brasiliense.

Bertola, P.; Manzini, E. (2006). Design multiverso: appunti di fenomenologia del design. Milano: POLI.design.

Borba, G. S. De; Reyes, P. (2009). Inovação orientada pelo design: A construção de uma organização voltada para a inovação. $4^{\circ}$ Congresso de Pesquisa em Design. Rio de Janeiro.

Celaschi, F.; Deserti, A. (2007). Design e innovazione: strumenti e pratiche per la ricercaapplicata. Roma: Carocci.

Celaschi, F. (2008a). L'uomo al centro del progetto. Torino: Editora Allemandi.

La carta di Torino. (2008b). $1^{\circ}$ Fórum Internacional de Escolas de Doutorados em Design "Changingthe Change". Torino: 12 Julho 2008.

Comte, P. (2010). Qu'est-Cequ'un workshop. In Enseignerle design? Org. Marie-Haude Caraes et Françoise Coeur, Citédu design.

Crossan, M.; Lane, H.; White, R. (1999). An organizational learning framework: from intuition to institution. Academy of Management Review, v. 24, n. 3, p. 522-537.

Flusser, V.; Cardoso, R. (Org.). (2007). O mundo codificado: por uma filosofia do design e da comunicação. São Paulo: Cosac Naify.

Foucault, M. (2006). Estética: literatura e pintura, música e cinema. 2. Ed. Rio de Janeiro: Forense Universitária.

Garcia, N. y Hermes, V. Publicado el 17 de Abril de 2009. Disponible en: http://casa.abril. com.br/materia/irmaos-campana-contam-sua-trajetoria-profissional-em-evento

Garvin, D. (1993). Building a learning organization. Harvard Business Review. v. 71, n. 4. Boston, p. 78-91.

Kim, D. (1993). The link between individual and organizational learning. Sloan Management Review, p. 37-50, Fall 1993.

Manzini, E. Artefatti. Verso una nuova ecologia dell'ambiente artificiale (1990). Milano: Domus Academy.

Marquardt, M. J. (1996). Building a learning organization: a system approach to quantum improvement and global success. New York: McGraw-Hill. 
Mintzberg, H. et al. (2006). O processo da estratégia: conceitos, contextos e casos selecionados. 4. ed. Porto Alegre: Bokman.

Moritz, S. (2004). Access to service design. London.

Senge, P. (1990). A quinta disciplina: arte, teoria e prática da organização de aprendizagem. São Paulo: Best-Seller, p. 352.

Scaletzky, C. \& Parode, F. P. (2008). Imagem e pesquisa Blue Sky no Design. XVI Convención científica de ingeniería y arquitetura. Cuba.

Tidd, J.; Bessant, J.; Pavitt, K. (2008). Gestão da Inovação. 3.ed. Porto Alegre: Bookman.

Veiga, I. P. A. (Org.) (2001). Projeto político-pedagógico da escola: uma construção possível. 23. ed. Campinas: Papirus.

Weick, K. (1995). Sensemaking in organizations. London.

Zurlo, F. (2006a). Design del sistema prodotto in Design multiverso: appuntidi fenomeno logia del design. Milano: POLI.design.

(2010b). Design Strategico. In: AA. VV,. Glispazi e learti, Volume IV, Opera XXI Secolo. Roma: Editora Enciclopédia Treccani.

Summary: Design as a phenomenological discipline is emerging in the current scenario, as a creating mechanism of synergies between companies and universities.

Many times, these relationships are based in an internal organizational processes review. In this context, design methodologies can establish new standards of organizational learning, creating forms of fluid and creative participation across sectors and skills. To illustrate this perspective, we present a learning model based on education through the practice of the reflective project, using the tool of the Workshop - important methodological tool for creative learning Strategic Design. Thus, this paper presents case studies of experiences of projective Workshops conducted in collaboration with the plastic shoe brand Melissa. The results were innovative, both in terms of organizational education, as in the sense of establishing new understanding of the design parameters for the designers of the brand.

Keywords: design methodologies - innovation - organizational learning - strategic design - workshop.

Resumo: O design como disciplina fenomenológica surge, no cenário atual, como mecanismo de criação de relações sinérgicas entre empresas e universidades. Muitas vezes, estas relações têm suas bases numa revisão dos processos internos das organizações. Neste contexto, as metodologias do design podem estabelecer novos parâmetros de aprendizagem organizacional, criando formas de participação fluida e criativa através de setores e competências. Para ilustrar essa perspectiva, apresentamos um modelo de aprendizagem baseado na educação através da prática do projeto/reflexivo, utilizando a ferramenta do Workshop, importante instrumento metodológico de aprendizagem criativa do Design Estratégico. Desta maneira. Este trabalho apresenta o estudo de casos das experiências 
projetivas dos Workshops feitos em colaboração com a marca de sapatos de plástico Melissa. Os resultados foram soluções inovadoras, tanto no sentido de educação organizacional, como no sentido de estabelecer novos parâmetros de compreensão do design para os projetistas da marca.

Palavras chave: aprendizagem organizacional - design estratégico - inovação - metodologias do design - workshop. 